This item was submitted to Loughborough's Research Repository by the author.

Items in Figshare are protected by copyright, with all rights reserved, unless otherwise indicated.

\title{
B-type natriuretic peptide molecular forms for risk stratification and prediction of outcome after acute myocardial infarction
}

PLEASE CITE THE PUBLISHED VERSION

https://doi.org/10.1016/j.ahj.2018.02.016

\section{PUBLISHER}

(C) Elsevier

VERSION

AM (Accepted Manuscript)

\section{PUBLISHER STATEMENT}

This work is made available according to the conditions of the Creative Commons Attribution-NonCommercialNoDerivatives 4.0 International (CC BY-NC-ND 4.0) licence. Full details of this licence are available at: https://creativecommons.org/licenses/by-nc-nd/4.0/

\section{LICENCE}

CC BY-NC-ND 4.0

\section{REPOSITORY RECORD}

Israr, M. Zubair, Liam M. Heaney, Leong L. Ng, and Toru Suzuki. 2019. "B-type Natriuretic Peptide Molecular Forms for Risk Stratification and Prediction of Outcome After Acute Myocardial Infarction”. figshare. https://hdl.handle.net/2134/33314. 
Full title: B-type Natriuretic Peptide Molecular Forms for Risk Stratification and Prediction of Outcome after Acute Myocardial Infarction

Short title: BNP Molecular Forms and Acute MI

Authors: M. Zubair Israr MSc ${ }^{1}$, Liam M. Heaney $\mathrm{PhD}^{1}$, Leong L. $\mathrm{Ng} \mathrm{MD}^{1} \uparrow$, Toru Suzuki $\mathrm{MD}^{1,2 * \dagger}$

$\dagger$ LLN and TS contributed equally to this manuscript

\section{Affiliations:}

${ }^{1}$ Department of Cardiovascular Sciences and NIHR Leicester Cardiovascular Biomedical Research Centre, University of Leicester, Glenfield Hospital, Leicester, LE3 9QP, UK

${ }^{2}$ Jichi Medical University, 3311-1 Yakushiji, Shimotsuke-shi, Tochigi-ken 329-0498, Japan

\section{Address for Correspondence:}

* Prof Toru Suzuki, Department of Cardiovascular Sciences and NIHR Leicester

Cardiovascular Biomedical Research Centre, University of Leicester, Glenfield Hospital, Leicester, LE3 9QP, United Kingdom. Email: ts263@le.ac.uk. Tel: (0044) 1162044741

\section{Keywords:}

biomarkers, BNP molecular forms, mass spectrometry, prognosis, risk prediction

\section{Conflicts of interest:}

The authors declare no conflicts of interest. 


\section{ABSTRACT}

Background: B-type natriuretic peptide (BNP) is known to be a risk marker following acute myocardial infarction (MI). More recently, truncated molecular forms of the BNP molecule have been identified, with the association of these forms and outcome in acute MI not known. The present study investigated their use as risk stratifying biomarkers of this condition.

Methods: BNP molecular forms (BNP 5-32, BNP 4-32 and BNP 3-32) were measured in plasma from 1,078 acute MI patients using immunocapture followed by MALDI-ToF-mass spectrometry. Associations of molecular forms with short-term and long-term adverse outcomes were assessed.

Results: BNP molecular forms were independent predictors of mortality/reinfarction, mortality/rehospitalization due to heart failure, and a composite of all events at 6 months, 1 year and 2 years and showed prognostic ability comparable with conventional BNP measurements $(P<0.001-0.026$ vs. N-terminal $[\mathrm{NT}]$-proBNP $P<0.001-0.020$, respectively). Reclassification analyses showed BNP molecular forms successfully reclassified patient risk when used in addition to the GRACE clinical risk score $(P \leq 0.005)$. BNP 5-32 showed utility as a secondary risk stratification biomarker when used in combination with the GRACE score and NT-proBNP by successful down-classification of high-risk patients.

Conclusions: BNP molecular forms were associated with poor prognosis at 6 months, 1 year and at 2 years in patients with acute MI. BNP 5-32 showed successful utility as a secondary marker in combination with NT-proBNP after GRACE scoring. This study suggests a potential role for BNP molecular forms in prognosis and risk stratification after acute MI. 


\section{INTRODUCTION}

B-type natriuretic peptide (BNP), and its N-terminal pro hormone (NT-proBNP), have been shown to be biomarkers of risk stratification and outcomes of patients following acute myocardial infarction (MI). Previous investigations have shown elevated levels of BNP or NT-proBNP are associated with in-hospital (1) and short-term (30-day) mortality (2), as well as mortality at extended follow-up periods of 4 years or more (3-5). From a pathophysiological standpoint, measured circulating levels of the BNP family of peptides have also shown associations with infarct size (6), degree of systolic dysfunction (7), development of heart failure (8) and cardiac death (9). Therefore, BNP peptides have been able to provide complementary clinical information beyond traditional MI biomarkers such as troponin and copeptin (3).

Truncated BNP fragments, or BNP molecular forms, are produced from proteolytic degradation of parent BNP molecules causing removal of end-chain amino acids groups, resulting in a range of fragmented peptides (e.g. BNP 3-29, 3-30, 4-29, 5-29 etc.) (10, 11). The most common molecular forms have been identified as BNP 5-32, BNP 4-32 and BNP 332 which possess the structure of BNP 1-32 with the removal of 4, 3 and 2 amino acids, respectively (10). The mechanistic pathways leading to the production of BNP molecular forms are not fully understood. Previous reports have suggested the involvement of multiple proteases including dipeptidyl peptidase-IV (BNP 3-32) (12), a neutral endopeptidase (BNP 5-32) processing of BNP 1-32 (13), and a corin-mediated cleavage of proBNP (BNP 4-32) (14). BNP molecular forms are understood to be reflective of conventional clinical measurements of circulating BNP, showing a greater association with results obtained from clinical pathology laboratories than the parent BNP 1-32 molecule measured in isolation (15). The biological impact of the degradation of the parent BNP 1-32 molecule currently lacks sufficient understanding. Previously, BNP molecular forms were reported to show no 
differences in their ability to generate cGMP when compared to BNP 1-32 (10). However, in vivo actions of BNP molecular form are suggested to exhibit reduced levels of natriuretic activity (10).

BNP molecular forms have been implicated in ischemic heart disease (16) and chronic HF (15), and more recently demonstrated as prognostic indicators for acute HF (17). For risk prediction following MI, troponin measurements are a robust and conventional way to stratify patients (18), with BNP peptides showing similarly successful application as prognostic biomarkers $(3,5,19)$. Furthermore, BNP has been suggested as a biomarker that reflects the severity of ischemia regardless of the extent of irreversibly injury, and therefore provides additive and important information in acute MI risk prediction (20).

The aims of the present study were to characterize circulating BNP molecular forms in acute MI patients and to investigate their prognostic ability to assist in risk stratification. Comparisons for the use of BNP molecular forms as prognostic indicators in acute MI were made with established measurements of circulating BNP (NT-proBNP and clinical BNP) and tested in combination with the established GRACE (Global Registry of Acute Coronary Events) risk prediction tool for MI (21). 


\section{MATERIALS AND METHODS}

\section{Study population}

One thousand and seventy-eight patients were admitted with acute MI to University Hospitals of Leicester, UK, between August 2004 and April 2007. Each patient consented (informed and written) to have blood samples taken and outcomes surveyed. This study was approved by the local ethics committee and adhered to the Declaration of Helsinki.

Diagnosis of acute MI was made on the basis that all patients had a cardiac troponin I (cTnI) concentration above the $99^{\text {th }}$ percentile, with at least one of the following: chest pain lasting >20 min or diagnostic serial electrocardiographic changes consisting of new pathological Q-waves or ST-segment and T-wave changes (22), excluding patients with malignancy, renal replacement therapy, or previous surgery within 1 month. Estimated glomerular filtration rate (eGFR) was calculated from the simplified Modification of Diet in Renal Disease formula (23). All patients received standard medical treatment and revascularization at the discretion of the attending physician.

\section{Venipuncture}

Peripheral intravenous blood samples were collected at time of discharge in prechilled tubes containing EDTA and aprotinin, and centrifuged at $1500 \mathrm{~g}$ for $20 \mathrm{~min}$ at $4{ }^{\circ} \mathrm{C}$. Plasma was aliquoted and stored at $-80^{\circ} \mathrm{C}$ until analysis. For analysis, samples were thawed at $37^{\circ} \mathrm{C}$, prepared, and analyzed immediately. 


\section{Measurement of BNP molecular forms}

Molecular forms of BNP were measured using a previously described immunocapture assay protocol with an analytical range of $20-3000 \mathrm{pg} / \mathrm{mL}(16,17)$. Briefly, molecular forms of BNP were isolated and captured from $100 \mu \mathrm{L}$ plasma using a monoclonal antibody raised against the ring region of BNP (Sekisui Medical Co., Tokyo, Japan) bound to magnetic beads (Fisher Scientific, Loughborough, UK). Captured forms were then eluted in $3 \mu \mathrm{L}$ of $0.1 \%$ trifluoroacetic acid (Sigma-Aldrich, Gillingham, UK), and $0.75 \mu \mathrm{L}$ spotted onto a metal target plate followed by $5 \mathrm{fmol} / \mu \mathrm{L}$ adrenocorticotropic hormone (ACTH) signal peptide (Sigma-Aldrich) and $10 \mathrm{mg} / \mathrm{mL}$ matrix (2,5-Dihydroxybenzoic acid and $\alpha$-Cyano-4hydroxycinnamic acid, LaserBio Labs, Sophia Antipolis, France) in a 1:1:1 ratio. Samples were detected using matrix assisted laser desorption ionization-time of flight-mass spectrometry (MALDI-ToF-MS) using an AXIMA Confidence (Shimadzu Corporation, Kyoto, Japan) in positive ion mode. A quality control (QC) sample consisting of all three BNP fragments at a concentration of $100 \mathrm{fmol} / \mathrm{mL}$ was performed daily to ensure analytical reproducibility. All peptides were sourced from Peptide Institute Inc., Osaka, Japan. The relative standard deviations of these QC analyses $(n=44)$ across the course of the study were $6.2 \%, 9.7 \%$ and $13.7 \%$ for BNP 5-32, BNP 4-32 and BNP 3-32, respectively. An example mass spectrum of the quality control sample showing peaks for BNP molecular forms can be found in the Supplementary File (Figure S1). Identifications of molecular BNP forms were confirmed by comparison of mass-to-charge ratio of detected ions to those detected in the positive control. For statistical analyses, mass spectral peak intensities of the BNP molecular forms were expressed as a ratio to the internal standard (ACTH). Samples where BNP molecular forms were not detectable had their result recorded as equal to the average value of the baseline noise:ACTH ratio (a value of 0.03 ). A visual indication of the baseline noise 
intensity in comparison to ACTH and BNP 5-32 at a value of 0.210 can be seen in the Supplemental File (Figure S2).

The antibodies used in this study allowed selective immunocapture of BNP molecular forms 5-32, 4-32 and 3-32. BNP 1-32, proBNP and other alternative BNP molecular forms were not detected for these samples using our experimental protocol. An example mass spectrum from a patient sample showing the presence of BNP molecular forms and absence of BNP 1-32 and proBNP can be found in the Supplemental File (Figure S3). NT-proBNP was measured using a sandwich immunoassay as described previously (5). A subset of patients with positive detection of BNP 5-32 $(n=617)$ were analyzed for clinical BNP levels using a point-of-care device (RapidPIA®, Sekisui Medical Co.).

\section{End points}

Primary outcomes were composites of all-cause mortality or reinfarction (death/MI), all-cause mortality or rehospitalization due to $\mathrm{HF}$ (death/HF), as well as death/MI and death/HF combined (MACE). Outcomes were measured in all patients for short-term (6 months) and long-term (1 year and 2 years) risk prediction.

Addition of BNP molecular forms to the GRACE score for outcomes at 6 months was tested for the end point of death/MI. End points were obtained by reviewing the local hospital databases and the Office of National Statistics Registry, and by telephone calls to patients, and those data were verified by reviewing medical records. One hundred percent follow-up was achieved. 


\section{Statistical analyses}

Statistical analyses were performed using IBM SPSS Statistics (V24, IBM Corp., Armonk, New York, USA). Spearman's rank-order correlations were calculated for molecular forms of BNP against NT-proBNP, clinical BNP and other traditional risk markers of acute MI and cardiovascular disease. Outcome prediction accuracies were assessed by calculating the area under the curve (AUC) of the Receiver Operator Characteristic (ROC) for BNP peptides across all end points. Cox proportional hazard analyses were performed to identify independent predictors of death/MI, death/HF and MACE at 6 months, 1 year and 2 years. Values for molecular forms of BNP, NT-proBNP and clinical BNP were log transformed. Continuous reclassification analyses (24) were used to assess the utility of adding BNP molecular forms and NT-proBNP to the GRACE score for risk assessment at 6 months. Decision tree analysis was performed using the $\chi^{2}$ automatic interaction detection (CHAID). A $P$-value $<0.05$ was deemed statistically significant.

\section{Sources of funding}

This work was supported by the Practical Research Project for Life-Style related Diseases including Cardiovascular Diseases and Diabetes Mellitus from Japan Agency for Medical Research and Development (AMED) (17ek0210011h0005), the Japan Heart Foundation, the University of Tokyo, the John and Lucille van Geest Foundation and the National Institute for Health Research Leicester Biomedical Research Centre. The authors are solely responsible for the design and conduct of this study, all study analyses, the drafting and editing of the paper and its final contents. 


\section{RESULTS}

\section{Patient characteristics}

Plasma samples from 1,078 patients admitted to hospital with acute MI were analyzed for the presence of BNP molecular forms. Mass spectral peaks for BNP molecular forms were detected in a total of $617(57.2 \%)$ samples. A breakdown of the measured end points and clinical demographics for the patient cohort is shown in Table I.

\section{Analyses with associated clinical measurements and outcomes}

Spearman's rank-order correlation analyses showed clinical variables that were associated with one or more of the BNP molecular forms to be blood urea, eGFR, age, cTnI at admission, heart rate, blood glucose, blood sodium and systolic blood pressure (Table II). BNP molecular forms were strongly correlated to each other $\left(r_{s}=0.798-0.935, P<0.001\right)$ and NT-proBNP levels $\left(r_{s}=0.591-0.640, P<0.001\right)$. BNP molecular forms were correlated with markers of renal dysfunction including blood urea $\left(r_{s}=0.267-0.272, P<0.001\right)$ and eGFR $\left(r_{s}\right.$ $=-0.323--0.343, P<0.001)$ but at a level that was modestly reduced when compared with NT-proBNP; for blood urea $\left(r_{s}=0.324, P<0.001\right)$ and eGFR $\left(r_{s}=-0.376, P<0.001\right)$. Patient samples with positive detection of BNP 5-32 showed correlation with clinical BNP measurements $\left[r_{s}=0.682,0.732\right.$ and 0.645 (all $P<0.001$ ) for BNP 5-32, 4-32 and 3-32, respectively] in accordance to previous reports (data not shown) (15). The accuracies for prediction of the investigated outcomes were tested by analyzing the AUC (c-statistic) of the ROC curve and are shown for all BNP molecular forms and NT-proBNP in Table III. 


\section{BNP molecular forms as predictors of death/MI}

To investigate the prognostic ability of BNP molecular forms for death/MI at 6 months, 1 year and 2 years, Cox survival analyses were conducted using a multivariable model adjusted for traditional cardiovascular disease risk factors. The risk factors included in the model were age, sex, systolic BP, Killip score >1, STEMI class, revascularization, medication at discharge (aspirin, $\beta$-blockers, ACE/ARB, statins), renal markers (eGFR and urea), admission cTnI levels and past histories of MI/angina, diabetes and hypertension. Independent abilities for the molecular forms of BNP and NT-proBNP to predict outcome were assessed by adding each marker to the base model. NT-proBNP was a univariate predictor of death/MI at 6 months, 1 year and at 2 years $(P<0.001)$, and retained independent prediction at these end points $(P \leq 0.020)$ when added to the base model (Table IV). Similarly, BNP molecular forms were univariate predictors of death/MI at 6 months, 1 year and at 2 years (all $P<0.001)$. When BNP molecular forms were added to the base model, they retained independent prediction at 6 months (BNP 5-32, 4-32, 3-32; $P=0.019, P=0.017, P$ $=0.026), 1$ year $(P=0.004, P=0.005, P=0.018)$ and at 2 years $(P=0.001, P=0.002, P=$ 0.008) showing comparable abilities to NT-proBNP (Table IV). Other independent predictors of outcome were age, urea and revascularization.

\section{BNP molecular forms as predictors of death/HF}

To investigate the prognostic ability of BNP molecular forms for death/HF at 6 months, 1 year and 2 years, Cox survival analyses were conducted using the same multivariate base model as described for death/MI. NT-proBNP was a univariate predictor of death/HF at 6 months, 1 year and at 2 years $(P \leq 0.001)$, and retained independent prediction at these end points $(P \leq 0.001)$ when added to the base model. BNP molecular forms were 
also significant univariate predictors of death/HF at 6 months, 1 year and at 2 years (all $P$ $<0.001)$. When added to the base model, BNP molecular forms retained independent prediction at 6 months (BNP 5-32, 4-32, 3-32; $P=0.026, P=0.007, P=0.002), 1$ year $(P=$ 0.007, $P=0.001, P=0.001)$ and at 2 years $(P=0.001, P<0.001, P<0.001)$ showing comparable prognostic abilities to NT-proBNP (Table IV). Other independent predictors of outcome were age, Killip score $>1$, STEMI class, systolic BP, $\beta$-blockers and statins on discharge and eGFR.

\section{BNP molecular forms as predictors of MACE}

NT-proBNP was a univariate predictor of MACE at 6 months, 1 year and at 2 years $(P<0.001)$, and retained independent prediction at these end points $(P \leq 0.005)$ when added to the previously defined base model. BNP molecular forms were also significant univariate predictors of MACE at 6 months, 1 year and at 2 years (all $P<0.001$ ). When BNP molecular forms were added to the model they retained independent prediction at 6 months (BNP 5-32, 4-32, 3-32; $P=0.017, P=0.011, P=0.005), 1$ year $(P=0.002, P=0.001, P=0.002)$ and at 2 years $(P<0.001, P<0.001, P<0.001)$ showing comparable abilities to NT-proBNP (Table IV). Other independent predictors of outcome were age, Killip score >1, STEMI class, revascularization, systolic $\mathrm{BP}$ and $\beta$-blockers on discharge.

\section{Comparison of BNP molecular forms against clinical BNP measurements}

Clinical BNP values were measured using a commercial assay in a subset of samples $(n=617)$ with detectable levels of BNP molecular forms. Cox proportional hazards regressions were performed using the described clinical base-model to understand the predictive ability of BNP molecular forms in comparison with clinical BNP. These analyses 
were performed for all described end points and are shown in Supplemental Table S1. BNP and BNP molecular forms showed similar predictive abilities across outcome measures and their respective time points. Interestingly, NT-proBNP was not able to predict outcome in this subset of patients for time points associated with the end point of Death/MI $(P \geq 0.092)$. This indicated a modest improvement in predictive ability for BNP and its molecular forms over NT-proBNP.

\section{Reclassification analyses}

Reclassification analyses were performed using the continuous net reclassification improvement index to assess the added value of BNP molecular forms and NT-proBNP to the current GRACE clinical risk score for outcome at 6 months. GRACE score values were available for a total of $921(85.4 \%)$ patients. Results showed that both NT-proBNP and BNP molecular forms showed a total improvement in reclassification when added to the GRACE score $(P \leq 0.005)$ (Table V). NT-proBNP and BNP 5-32 were able to successfully downclassify risk in patients without an event and up-classify those with an event, providing a successful overall reclassification of patients for adverse event risk $(P<0.001)$. Whilst BNP 4-32 and BNP 3-32 were able to successfully reclassify overall patient risk $(P=0.005$ and $P$ $=0.003$, respectively), the effect was weakened in both molecular forms through an inability to successfully up-classify patients with events (Table V).

\section{Decision tree analysis}

Decision tree analysis was performed to assess the utility of using BNP 5-32 and NTproBNP as secondary risk stratification markers after applying the GRACE score for risk prediction (death/MI at 6 months). Results showed that using GRACE as the primary 
classifier, a score >138 and NT-proBNP concentration above $1834.4 \mathrm{pmol} / \mathrm{L}$ identified the highest risk group $(n=143)$, with a group event risk of $39.2 \%$. In contrast, a GRACE score of $\leq 138$ and BNP 5-32 value of $\leq 0.240$ identified the lowest risk group ( $n=444$ ), with a group event risk of $6.5 \%$ showing successful down-stratification of patient risk. Kaplan-Meier survival analysis for the outcome of death/MI at 6 months with patients stratified into three groups, as defined by the decision tree analysis, showed a significant increase in risk across the groups $(P \leq 0.001)$ with a fold-increase in risk of 2.6 and 9.1 for the middle- and high-risk groups when compared to the low-risk reference group $(P<0.001)$, respectively (Figure 1). These data support the combined use of NT-proBNP and BNP molecular forms as secondary risk stratification biomarkers for acute MI patients in combination with established clinical risk algorithms. 


\section{DISCUSSION}

This study reports that molecular forms of BNP are associated with poor outcome in patients hospitalized with acute MI, and the utility of these molecular forms in clinical risk prediction. The results showed that molecular BNP forms 5-32, 4-32 and 3-32 were all independently able to predict death/MI, death/HF and MACE at 6 months, 1 year and at 2 years after adjustment for traditional clinical and physiological factors. These prognostic qualities were comparable to conventional measurements of circulating BNP through analysis of plasma NT-proBNP and clinical BNP concentrations. Although clinical measurements of BNP have been shown as useful in prediction of outcome after acute MI for long-term survival (3), this is the first study to report the prognostic capabilities of BNP molecular forms in an acute MI cohort. These findings extend from previous evidence for the utility of BNP molecular forms in acute HF prognosis (17).

Similarly to results previously published in acute HF (17), BNP 5-32 exhibited mildly superior risk prediction capabilities when compared to alternative molecular forms. The BNP molecular forms were successfully able to predict outcome at all measured endpoints, with an improvement in prognostic qualities with extended follow-up periods. This suggests that although suitable for shorter-term prediction, BNP molecular forms offer improved information for risk stratification over time.

Reclassification analyses were performed after combining BNP molecular forms with the established GRACE risk prediction tool for death/MI at 6 months and showed an added benefit for the use of BNP molecular forms. BNP 5-32 was the superior molecular form with the ability to reclassify patients for both increased and decreased risk after classification by the GRACE score and was comparable to results obtained for NT-proBNP. In contrast, although BNP 4-32 and BNP 3-32 were successful in an overall reclassification of patient 
risk, this was centered on their strong ability to down-classify risk with both molecular forms reporting negative net reclassification indices for the up-classification of patient risk. Reclassification analyses have demonstrated the ability to offer great sensitivity in highlighting an improvement for the inclusion of an additional variable to a previously calculated value (e.g. clinical risk score) (25). Further improvements in sensitivity are gained through the use of continuous category-free reclassification (24), as performed in this investigation. However, it has also been argued that reclassification analyses demonstrate an oversensitivity for risk stratification (26), and therefore our data should be interpreted in combination with the other statistical tests performed.

BNP 5-32, as the most discriminatory of molecular forms and its improved detection in patient samples, was taken forward into decision tree analysis and used in conjunction with NT-proBNP to define risk stratification following GRACE scoring. BNP 5-32 identified patients at lower risk after initial GRACE screening with NT-proBNP highlighting those at highest risk, generating patient groupings that showed a stepped increase in risk. A GRACE score of 138 identifies patients in the high-risk category for both non-STEMI and STEMI (GRACE score $\geq 119$ for non-STEMI and $\geq 128$ for STEMI) (27). These data suggest the beneficial use for combined analyses of BNP family peptides in enhancing risk prediction of acute MI patients. In this instance, BNP 5-32 and NT-proBNP were selected as complementary secondary biomarkers to a clinical risk algorithm for risk stratification at 6 months, demonstrating the advantages of BNP molecular forms in addition to current models of single BNP peptide analyses. Previous studies have shown NT-proBNP to add prognostic value to the GRACE score in elderly patients with acute MI (28) whilst also being able to predict early and late mortality in risk stratification after acute coronary syndromes (29).

Although this study provides information regarding BNP molecular forms in acute MI, measurement of these forms does not add tremendously to the significance of natriuretic 
peptides measured in a conventional way. The present analyses did not take into account proBNP levels as they were not measured using the described mass spectrometric assay which might influence the effects of BNP molecular forms in risk prediction.

In conclusion, the present study shows circulating BNP molecular forms are associated with poor prognosis (death/MI, death/HF and MACE) at 6 months, 1 year and at 2 years. When used in combination with NT-proBNP, BNP 5-32 showed utility as a secondary risk stratification biomarker in identifying low-risk patients for outcome at 6 months after initial categorization with the GRACE score. This study supports the added value of BNP molecular forms to contemporary BNP measurements in prognosis and risk stratification of acute cardiovascular hospitalizations. Currently, the assays to measure BNP molecular forms are research-focused and further developments of quantitative multiplex assays that allow the measurement of multiple BNP forms in a single analysis would be advantageous for clinical use. Importantly, care must be taken for future study of these molecular forms for the correct collection and storage of samples that is paramount to maintain sample integrity through the use of protease inhibitors (e.g. aprotinin) and ultra-cold storage conditions $\left(-80{ }^{\circ} \mathrm{C}\right)$. Furthermore, these data show value for further investigations into the dynamics and kinetics of BNP degradation as well as BNP pathway responses to disease management strategies. 


\section{ACKNOWLEDGEMENTS}

The authors are grateful to Sekisui Medical Co. for provision of antibodies and RapidPIA® BNP kits.

\section{DISCLOSURES}

The authors have no disclosures. 


\section{REFERENCES}

(1) Scirica, B.M., Kadakia, M.B., de Lemos, J.A., et al. Association between Natriuretic Peptides and Mortality among Patients Admitted with Myocardial Infarction: A Report from the ACTION Registry®-GWTG ${ }^{\mathrm{TM}}$. Clin Chem. 2013;59;1205-14. DOI: 10.1373/clinchem.2012.198556

(2) Mega, J.L., Morrow, D.A., De Lemos, J.A., et al. B-type natriuretic peptide at presentation and prognosis in patients with ST-segment elevation myocardial infarction: an ENTIRE-TIMI-23 substudy. $J$ Am Coll Cardiol. 2004;44:335-9. DOI: 10.1016/j.jacc.2004.04.033

(3) Omland, T., Aakvaag, A., Bonarjee, V.V., et al. Plasma brain natriuretic peptide as an indicator of left ventricular systolic function and long-term survival after acute myocardial infarction. Circulation. 1996;93:1963-9. DOI 10.1161/01.CIR.93.11.1963

(4) Richards AM, Nicholls MG, Espiner EA, et al. B-type natriuretic peptides and ejection fraction for prognosis after myocardial infarction. Circulation. 2003;107:2786-92. DOI: 10.1161/01.CIR.0000070953.76250.B9

(5) Omland T, Persson A, Ng L, et al. N-terminal pro-B-type natriuretic peptide and longterm mortality in acute coronary syndromes. Circulation. 2002;106:2913-8. DOI: 10.1161/01.CIR.0000041661.63285.AE

(6) Mayr A, Mair J, Schocke M, et al. Predictive value of NT-pro BNP after acute myocardial infarction: relation with acute and chronic infarct size and myocardial function. Int J Cardiol. 2011;147:118-23. DOI 10.1016/j.ijcard.2009.09.537 
(7) Gunes Y, Okcun B, Kavlak E, Erbas C, Karcier S. Value of brain natriuretic peptide after acute myocardial infarction/Akut miyokard infarktusu sonrasi beyin natriuretik peptid'in degeri. Anadolu Kardiyol Derg. 2008;8:182-8.

(8) Darbar D, Davidson NC, Gillespie N, et al. Diagnostic value of B-type natriuretic peptide concentrations in patients with acute myocardial infarction. Am J Cardiol. 1996;78:284-7. DOI: 10.1016/S0002-9149(96)00278-0

(9) Fazlinezhad A, Rezaeian MK, Yousefzadeh H, et al. Plasma brain natriuretic peptide (BNP) as an indicator of left ventricular function, early outcome and mechanical complications after acute myocardial infarction. Clin Med Insights Cardiol. 2011;5:77. DOI: 10.4137/CMC.S7189

(10) Niederkofler EE, Kiernan UA, O'rear J, et al. Detection of endogenous B-type natriuretic peptide at very low concentrations in patients with heart failure. Circ Heart Fail. 2008;4:25864 DOI: 10.1161/CIRCHEARTFAILURE.108.790774

(11) Zhang S, Raedschelders K, Santos M, Van Eyk JE. Profiling B-Type Natriuretic Peptide Cleavage Peptidoforms in Human Plasma by Capillary Electrophoresis with Electrospray Ionization Mass Spectrometry. J Proteome Res. 2017;16:4515-22. DOI: 10.1021/acs.jproteome.7b00482

(12) Brandt I, Lambeir AM, Ketelslegers JM, et al. Dipeptidyl-peptidase IV converts intact B-type natriuretic peptide into its des-SerPro form. Clin Chem. 2006;52:82-7. DOI: 10.1373/clinchem.2005.057638

(13) Kenny AJ, Bourne A, Ingram J. Hydrolysis of human and pig brain natriuretic peptides, urodilatin, C-type natriuretic peptide and some C-receptor ligands by endopeptidase-24.11. Biochem J. 1993;291:83- 8. DOI: 10.1042/bj2910083 
(14) Semenov AG, Tamm NN, Seferian KR, et al. Processing of pro-B-type natriuretic peptide: furin and corin as candidate convertases. Clin Chem. 2010;56:1166 -76. DOI: 10.1373/clinchem.2010.143883

(15) Miller WL, Phelps MA, Wood CM, et al. Comparison of mass spectrometry and clinical assay measurements of circulating fragments of B-type natriuretic peptide in patients with chronic heart failure. Circ Heart Fail. 2011;4:355-60. DOI: 10.1161/CIRCHEARTFAILURE. 110.960260

(16) Fujimoto H, Suzuki T, Aizawa K, et al. Processed B-type natriuretic peptide is a biomarker of postinterventional restenosis in ischemic heart disease. Clin Chem. 2013;59:1330-7. DOI: 10.1373/clinchem.2013.203406

(17) Suzuki T, Israr MZ, Heaney LM, et al. Prognostic role of molecular forms of B-type natriuretic peptide in acute heart failure. Clin Chem. 2017;63:880-6. DOI: 10.1373/clinchem.2016.265140

(18) Lindahl B, Toss H, Siegbahn A, Venge P, Wallentin L. Markers of myocardial damage and inflammation in relation to long-term mortality in unstable coronary artery disease. FRISC Study Group. Fragmin during Instability in Coronary Artery Disease. New Engl J Med. 2000;343:1139-47. DOI: 10.1056/NEJM200010193431602

(19) James SK, Lindahl B, Siegbahn A, et al. N-terminal pro-brain natriuretic peptide and other risk markers for the separate prediction of mortality and subsequent myocardial infarction in patients with unstable coronary artery disease: a Global Utilization of Strategies To Open occluded arteries (GUSTO)-IV substudy. Circulation. 2003;108:275-81. DOI: 10.1161/01.CIR.0000079170.10579.DC 
(20) Morrow DA, Braunwald E. Future of biomarkers in acute coronary syndromes: moving toward a multimarker strategy. Circulation. 2003;108:250-2. DOI: 10.1161/01.CIR.0000078080.37974.D2

(21) Eagle KA, Lim MJ, Dabbous $\mathrm{OH}$, et al. A validated prediction model for all forms of acute coronary syndrome: estimating the risk of 6-month postdischarge death in an international registry. JAMA. 2004;291:2727-33. DOI: 10.1001/jama.291.22.2727

(22) Thygesen K, Alpert JS, White HD, et al. Universal definition of myocardial infarction: Kristian Thygesen, Joseph S. Alpert and Harvey D. White on behalf of the Joint ESC/ACCF/AHA/WHF Task Force for the Redefinition of Myocardial Infarction. Circulation. 2007;28:2525-38. DOI: 10.1161/CIRCULATIONAHA.107.187397

(23) Smilde TD, Van Veldhuisen DJ, Navis G, et al. Drawbacks and prognostic value of formulas estimating renal function in patients with chronic heart failure and systolic dysfunction.

Circulation. 2006;114:1572-80.

DOI: 10.1161/CIRCULATIONAHA.105.610642

(24) Pencina MJ, Steyerberg EW, D’Agostino RB Sr. Extensions of net reclassification improvement calculations to measure usefulness of new biomarkers. Stat Med. 2011;30:11-21. DOI: $10.1002 / \operatorname{sim} .4085$

(25) Pencina MJ, D'Agostino RB Sr, D'Agostino RB Jr, Vasan RS. Evaluating the added predictive ability of a new marker: from area under the ROC curve to reclassification and beyond. Stat Med. 2008;27:157-22. DOI: 10.1002/sim.2929

(26) Pepe MS, Fan J, Feng Z, Gerds T, Hilden J. The Net Reclassification Index (NRI): a Misleading Measure of Prediction Improvement Even with Independent Test Data Sets. Stat Biosci. 2015;7:282-95. DOI: 10.1007/s12561-014-9118-0 
(27) Global Registry of Acute Coronary Events. GRACE risk table. https://www.outcomesumassmed.org/GRACE/grace risk table.aspx (Accessed May 2017).

(28) Lorgis L, Zeller M, Dentan G, et al. Prognostic value of N-terminal pro-brain natriuretic peptide in elderly people with acute myocardial infarction: prospective observational study. BMJ. 2009;338:b1605. DOI: 10.1136/bmj.b1605

(29) Khan SQ, Narayan $\mathrm{H}, \mathrm{Ng} \mathrm{KH}$, et al. N-terminal pro-B-type natriuretic peptide complements the GRACE risk score in predicting early and late mortality following acute coronary syndrome. Clin Sci. 2009;117:31-9. DOI: 10.1042/CS20080419 
Table I Patient demographics for acute myocardial infarction admissions.

\begin{tabular}{|c|c|}
\hline Age (yrs) & $67(57-77)$ \\
\hline Male & $72 \%$ \\
\hline Systolic BP (mmHg) & $136(120-151)$ \\
\hline Diastolic BP (mmHg) & $77(68-88)$ \\
\hline Heart rate (beats/min) & $75(63-95)$ \\
\hline Past history MI/angina (\%) & $33 \%$ \\
\hline Past history hypertension (\%) & $52 \%$ \\
\hline Past history diabetes & $23 \%$ \\
\hline Past history HF & $4 \%$ \\
\hline Aspirin on discharge (\%) & $84 \%$ \\
\hline$\beta$-blockers on discharge $(\%)$ & $81^{\circ}$ \\
\hline ACE/ARB on discharge (\%) & $84 \%$ \\
\hline Statins on discharge $(\%)$ & $89 \%$ \\
\hline Killip score $>1(\%)$ & $41 \%$ \\
\hline ST elevation MI (\%) & $47 \%$ \\
\hline Revascularization (\%) & $26 \%$ \\
\hline Glucose (mmol/L) & $7.5(6.3-9.9)$ \\
\hline Troponin (cTnI) (ng/mL) & $3.6(1.0-12.1)$ \\
\hline Urea (mg/dL) & $17(14-22)$ \\
\hline $\mathrm{eGFR}\left(\mathrm{mL} / \mathrm{min} / 1.73 \mathrm{~m}^{2}\right)$ & $66(53-78)$ \\
\hline $\mathrm{Na}^{+}(\mathrm{mmol} / \mathrm{L})$ & $138(136-140)$ \\
\hline $\mathrm{K}^{+}(\mathrm{mmol} / \mathrm{L})$ & $4.2(4.0-4.6)$ \\
\hline NT-proBNP (pmol/L) & $813(260-2199)$ \\
\hline GRACE score (22) & $120(96-143)$ \\
\hline BNP 5-32 & $0.2(0.03-0.4)$ \\
\hline BNP 4-32 & $0.04(0.03-0.2)$ \\
\hline BNP 3-32 & $0.04(0.03-0.2)$ \\
\hline
\end{tabular}

\section{Endpoints}

\section{6 months}

Death/MI 161

$\begin{array}{ll}\text { Death/HF } & 146\end{array}$ 
1 year

Death/MI 203

Death/HF 179

MACE 260

2 years

Death/MI 232

Death/HF 200

MACE 292

Data are reported as median (interquartile range) for continuous variables and as a $\%$ for categorical data.

Molecular BNP forms are reported as a ratio of mass spectral peak signal intensity against an internal reference standard.

BNP, B-type natriuretic peptide; BP, blood pressure; eGFR, estimated glomerular filtration rate; GRACE, Global Registry of Acute Coronary Events; HF, heart failure; MACE, major adverse cardiac event; MI, myocardial infarction, NT-proBNP, N-terminal pro B-type natriuretic peptide; NYHA, New York Heart Association 
Table II BNP molecular forms and associated clinical factors.

\begin{tabular}{ccccccccc}
\hline & \multicolumn{2}{c}{ BNP 5-32 } & \multicolumn{2}{c}{ BNP 4-32 } & \multicolumn{2}{c}{ BNP 3-32 } & \multicolumn{2}{c}{ NT-proBNP } \\
& $\boldsymbol{r}_{\boldsymbol{s}}$ & $\boldsymbol{P}$ Value & $\boldsymbol{r}_{\boldsymbol{s}}$ & $\boldsymbol{P}$ Value & $\boldsymbol{r}_{\boldsymbol{s}}$ & $\boldsymbol{P}$ Value & $\boldsymbol{r}_{\boldsymbol{s}}$ & $\boldsymbol{P}$ Value \\
\hline Urea & 0.267 & $<0.001$ & 0.272 & $<0.001$ & 0.271 & $<0.001$ & 0.324 & $<0.001$ \\
eGFR & -0.323 & $<0.001$ & -0.343 & $<0.001$ & -0.329 & $<0.001$ & -0.376 & $<0.001$ \\
Age & 0.382 & $<0.001$ & 0.396 & $<0.001$ & 0.386 & $<0.001$ & 0.450 & $<0.001$ \\
Troponin (cTnI) & 0.183 & $<0.001$ & 0.116 & $<0.001$ & 0.111 & $<0.001$ & 0.196 & $<0.001$ \\
Heart Rate & 0.186 & $<0.001$ & 0.213 & $<0.001$ & 0.197 & $<0.001$ & 0.144 & $<0.001$ \\
Blood Glucose & 0.195 & $<0.001$ & 0.157 & $<0.001$ & 0.149 & $<0.001$ & 0.222 & $<0.001$ \\
Blood Sodium & -0.088 & 0.006 & -0.080 & 0.012 & -0.064 & 0.043 & -0.176 & $<0.001$ \\
Systolic BP & -0.074 & 0.020 & -0.055 & 0.086 & -0.057 & 0.073 & -0.094 & 0.004 \\
BNP 5-32 & & & 0.803 & $<0.001$ & 0.798 & $<0.001$ & 0.640 & $<0.001$ \\
BNP 4-32 & & & & & 0.935 & $<0.001$ & 0.593 & $<0.001$ \\
BNP 3-32 & & & & & & & 0.591 & $<0.001$ \\
\hline
\end{tabular}

BNP, B-type natriuretic peptide; BP, blood pressure; eGFR, estimated glomerular filtration rate; NT-proBNP, N-terminal proBNP; $r_{s}$, Spearman's rho 
Table III Area under the curve (AUC) for the Receiver Operator Characteristic to assess the accuracy of outcome prediction of BNP molecular forms and NT-proBNP for outcomes of all-cause mortality or reinfarction (death/MI), all-cause mortality or rehospitalization due to $\mathrm{HF}$ (death/HF) and a composite of all events (MACE) at 6 months, 1 year and 2 years. Results displayed as AUC (95\% CI) $P$ value.

\begin{tabular}{llll}
\hline & Death/MI & Death/HF & MACE \\
\hline BNP 5-32 & & & \\
6 months & $0.66(0.61-0.70), P<0.001$ & $0.71(0.66-0.76), P<0.001$ & $0.67(0.63-0.71), P<0.001$ \\
1 year & $0.66(0.62-0.70), P<0.001$ & $0.71(0.66-0.75), P<0.001$ & $0.67(0.63-0.71), P<0.001$ \\
2 years & $0.66(0.62-0.70), P<0.001$ & $0.71(0.67-0.75), P<0.001$ & $0.67(0.63-0.70), P<0.001$ \\
BNP 4-32 & $0.63(0.58-0.68), P<0.001$ & $0.70(0.65-0.75), P<0.001$ & $0.65(0.60-0.69), P<0.001$ \\
6 months & $0.64(0.59-0.68), P<0.001$ & $0.70(0.66-0.75), P<0.001$ & $0.65(0.61-0.69), P<0.001$ \\
1 year & $0.64(0.59-0.68), P<0.001$ & $0.70(0.66-0.74), P<0.001$ & $0.65(0.61-0.69), P<0.001$ \\
2 years & $0.63(0.58-0.68), P<0.001$ & $0.70(0.65-0.75), P<0.001$ & $0.65(0.60-0.69), P<0.001$ \\
BNP 3-32 & $0.63(0.59-0.68), P<0.001$ & $0.70(0.65-0.74), P<0.001$ & $0.65(0.60-0.69), P<0.001$ \\
6 months & $0.63(0.59-0.68), P<0.001$ & $0.70(0.66-0.75), P<0.001$ & $0.65(0.61-0.68), P<0.001$ \\
1 year & & & \\
2 years & & & \\
NT-proBNP & & & \\
6 months & $0.69(0.64-0.73), P<0.001$ & $0.78(0.74-0.82), P<0.001$ & $0.71(0.67-0.75), P<0.001$ \\
1 year & $0.68(0.64-0.72), P<0.001$ & $0.77(0.73-0.81), P<0.001$ & $0.70(0.67-0.74), P<0.001$ \\
2 years & $0.68(0.64-0.72), P<0.001$ & $0.77(0.73-0.80), P<0.001$ & $0.70(0.66-0.73), P<0.001$ \\
\hline
\end{tabular}


Table IV Independent prediction abilities of BNP molecular forms and NT-proBNP using multivariate Cox survival analyses for outcomes of all-cause mortality or reinfarction (death/MI), all-cause mortality or rehospitalization due to HF (death/HF) and a composite of all events (MACE) at 6 months, 1 year and 2 years. Results displayed as HR (95\% CI) $P$ value.

\begin{tabular}{|c|c|c|c|}
\hline & Death/MI & Death/HF & MACE \\
\hline \multicolumn{4}{|l|}{ BNP 5-32 } \\
\hline 6 months & $1.55(1.07-2.23), P=0.019$ & $1.55(1.05-2.28), P=0.026$ & $1.46(1.07-1.99), P=0.017$ \\
\hline 1 year & $1.62(1.17-2.24), P=0.004$ & $1.60(1.13-2.27), P=0.007$ & $1.55(1.18-2.05), P=0.002$ \\
\hline 2 years & $1.70(1.26-2.30), P=0.001$ & $1.78(1.28-2.47), P=0.001$ & $1.63(1.25-2.11), P<0.001$ \\
\hline \multicolumn{4}{|l|}{ BNP 4-32 } \\
\hline 6 months & $1.64(1.09-2.47), P=0.017$ & $1.74(1.16-2.61), P=0.007$ & $1.55(1.11-2.18), P=0.011$ \\
\hline 1 year & $1.67(1.17-2.39), P=0.005$ & $1.86(1.29-2.67), P=0.001$ & $1.67(1.23-2.26), P=0.001$ \\
\hline 2 years & $1.71(1.22-2.39), P=0.002$ & $1.96(1.39-2.75), P<0.001$ & $1.69(1.27-2.25), P<0.001$ \\
\hline \multicolumn{4}{|l|}{ BNP 3-32 } \\
\hline 6 months & $1.58(1.06-2.37), P=0.026$ & $1.88(1.25-2.82), P=0.002$ & $1.63(1.16-2.29), P=0.005$ \\
\hline 1 year & $1.54(1.08-2.21), P=0.018$ & $1.88(1.31-2.72), P=0.001$ & $1.64(1.21-2.32), P=0.002$ \\
\hline 2 years & $1.58(1.13-2.21), P=0.008$ & $2.06(1.46-2.91), P<0.001$ & $1.70(1.28-2.27), P<0.001$ \\
\hline \multicolumn{4}{|c|}{ NT-proBNP } \\
\hline 6 months & $1.62(1.08-2.41), P=0.019$ & $2.24(1.40-3.59), P=0.001$ & $1.72(1.21-2.45), P=0.002$ \\
\hline 1 year & $1.50(1.07-2.12), P=0.019$ & $2.02(1.35-3.02), P=0.001$ & $1.54(1.14-2.07), P=0.005$ \\
\hline 2 years & $1.44(1.06-1.96), P=0.020$ & $2.15(1.47-3.14), P<0.001$ & $1.47(1.12-1.93), P=0.005$ \\
\hline
\end{tabular}

Models adjusted for: age, sex, PH (past history) MI/angina, Killip score $>1$, estimated glomerular filtration rate, $\mathrm{PH}$ diabetes, troponin at admission (cTnI), ST elevation MI, revascularization, urea, systolic $\mathrm{BP}, \mathrm{PH}$ hypertension, aspirin on discharge, $\beta$-blockers on discharge, ACE/ARB on discharge, statins on discharge

BNP; B-type natriuretic peptide; HF, heart failure; MACE, major adverse cardiac event; NTproBNP, N-terminal proBNP 
Table V Reclassification analyses for all-cause mortality or reinfarction at 6 months using continuous reclassification showing the net reclassification index (NRI) of adding BNP molecular forms and NT-proBNP to the classification using the GRACE clinical risk score

\begin{tabular}{lcll}
\hline & Endpoint & NRI $(\mathbf{9 5} \%$ CI $)$ & P Value \\
\hline BNP 5-32 & No & $12.2(5.3-19.1)$ & 0.001 \\
Yes & $21.2(4.4-37.9)$ & 0.013 \\
BNP 4-32 & Total & $33.4(15.3-51.5)$ & $<0.001$ \\
& No & $26.9(20-33.9)$ & $<0.001$ \\
Yes & $-0.7(-17.5-33.9)$ & NS \\
BNP 3-32 & Total & $26.2(8.1-44.3)$ & 0.005 \\
& No & $31.2(24.3-38.1)$ & $<0.001$ \\
& Yes & $-.36(-20.4-13.1)$ & NS \\
NT-proBNP & Total & $27.5(9.4-45.6)$ & 0.003 \\
& No & $9.8(2.8-16.8)$ & 0.006 \\
& Yes & $30.9(14.1-47.7)$ & $<0.001$ \\
& Total & $40.7(22.5-58.9)$ & $<0.001$ \\
\hline
\end{tabular}

95\% CI, 95\% confidence intervals; BNP; B-type natriuretic peptide; NRI, net reclassification index; NT-proBNP, N-terminal proBNP 


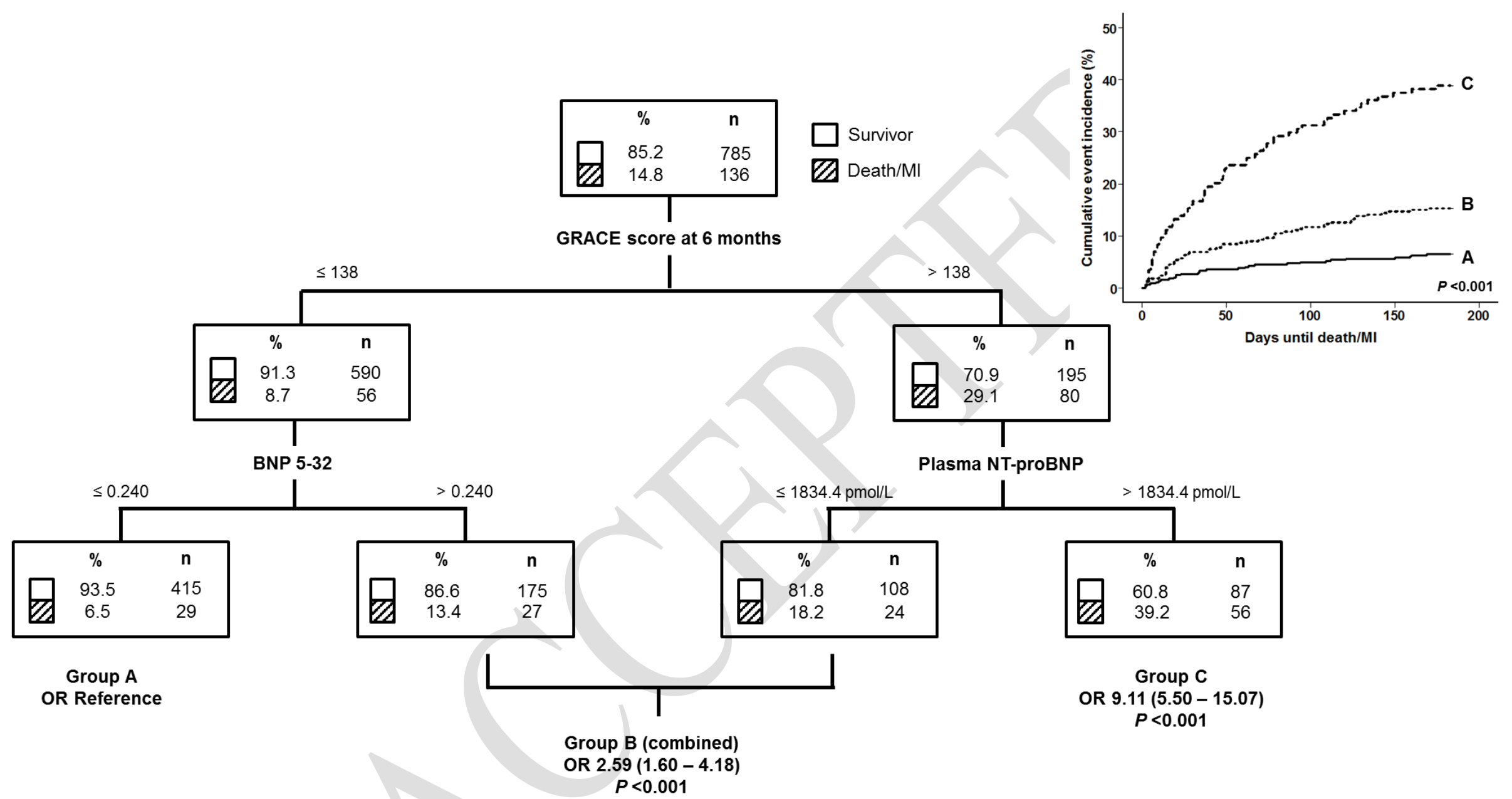

Figure 1 Decision tree showing risk stratification for the combined use of the GRACE clinical risk score for all-cause mortality or reinfarction (death/MI at 6 months), BNP 5-32 and NT-proBNP and cumulative event incidence of risk groups (inset).

BNP, B-type natriuretic peptide; NT-proBNP, N-terminal proBNP; OR, odds ratio 
SUPPLEMENTAL MATERIAL

B-type natriuretic peptide molecular forms for Risk Stratification and Prognosis after Acute Myocardial Infarction

M. Zubair Israr, Liam M. Heaney, Leong L. Ng, Toru Suzuki 
BNP 5-32, BNP 4-32 and BNP 3-32 (100 fmol/mL)

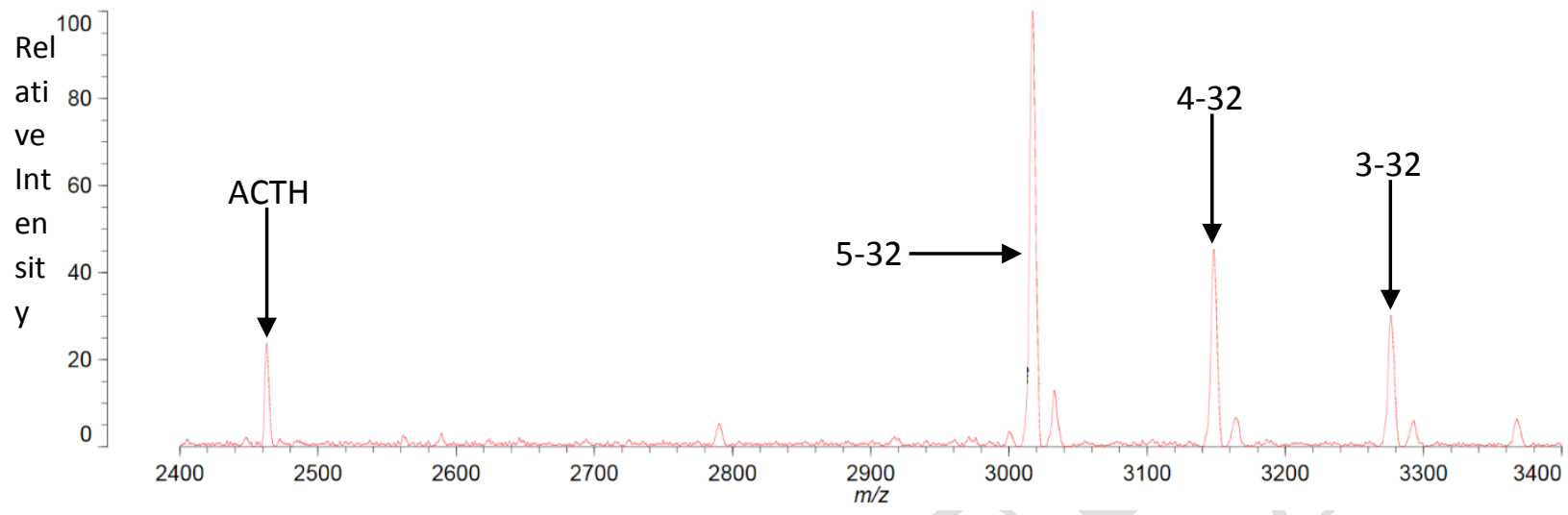

ACTH, Adrenocorticotrophic hormone; BNP, B-type natriuretic peptide, $\mathrm{m} / \mathrm{z}$, mass-to-charge ratio

\section{Supplemental Figure S2}

Example spectra showing the ACTH signal, baseline noise and BNP 5-32 ratios.

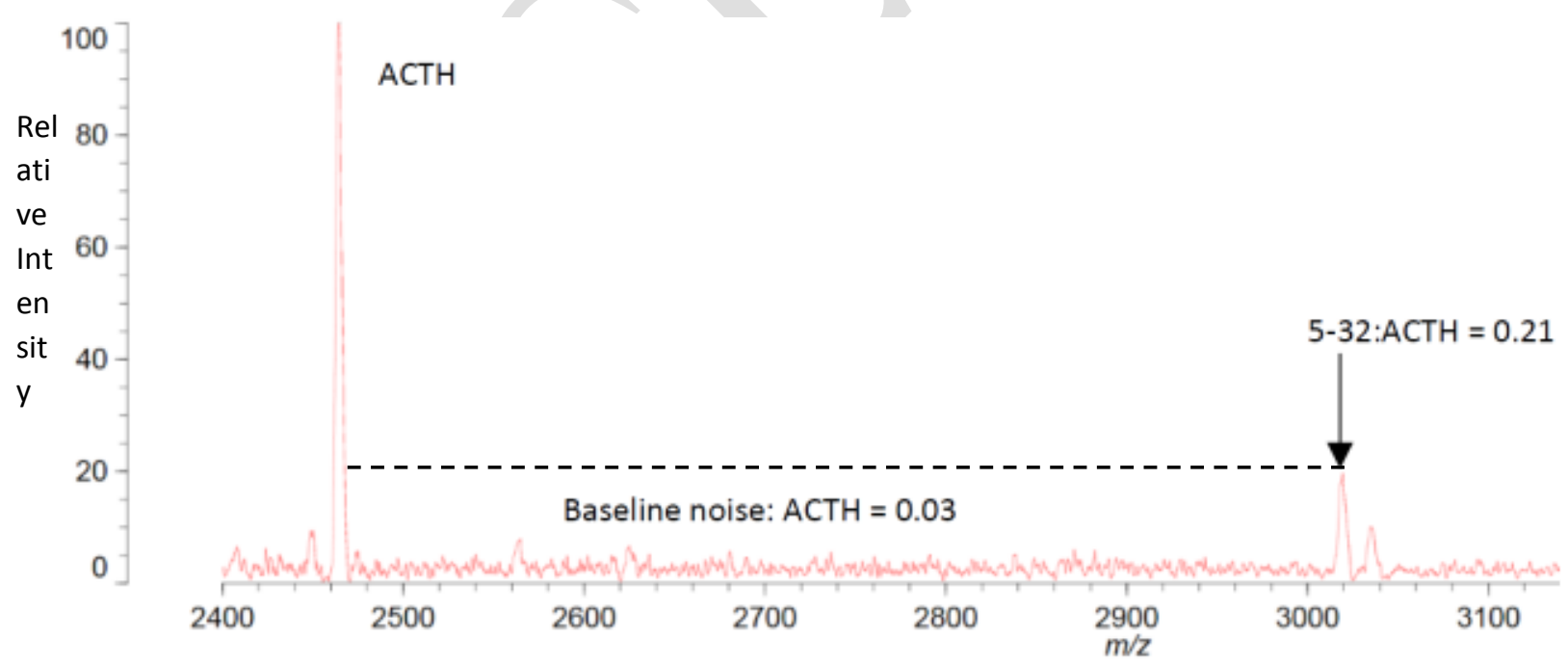

ACTH, Adrenocorticotrophic hormone; BNP, B-type natriuretic peptide, $m / z$, mass-to-charge ratio 
Supplemental Figure S3

Example spectra confirmation of circulating molecular forms

of BNP detected alongside ACTH signal peptide in acute MI plasma, and non-detection of BNP 1-32 and proBNP

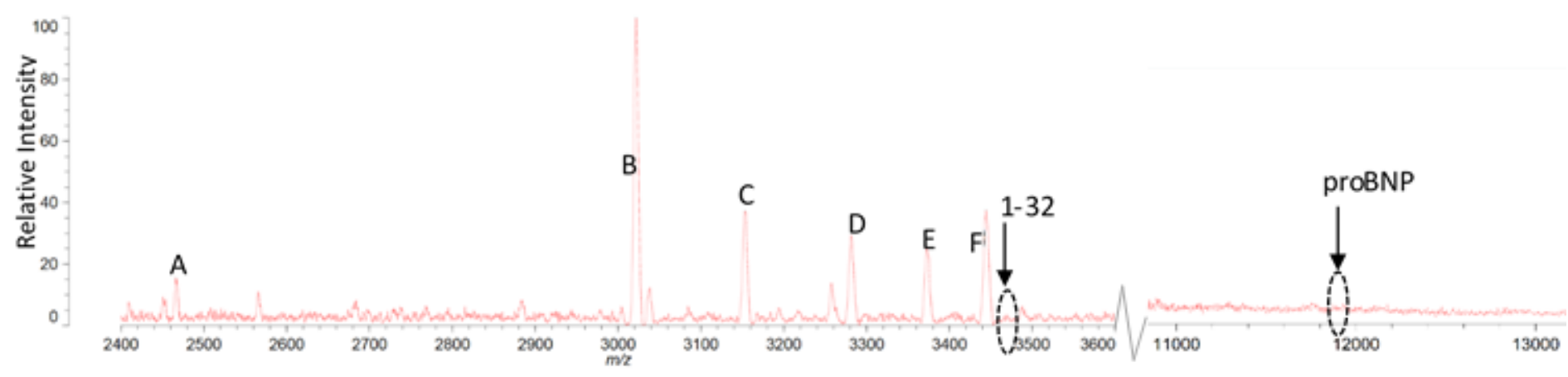

\begin{tabular}{lll}
\hline & Expected mass & Actual mass \\
\hline A - ACTH & 2464.20 & 2466.84 \\
B - BNP 5-32 & 3022.51 & 3021.92 \\
C - BNP 4-32 & 3153.71 & 3153.31 \\
D - BNP 3-32 & 3281.88 & 3281.24 \\
E & Unknown peak, detected in previously reported studies. & 3373.06 \\
F & Unknown peak, detected in previously reported studies. & 3444.52 \\
1-32 & 3466.08 & Undetected \\
proBNP & 11905.55 & Undetected \\
\hline
\end{tabular}

$\mathrm{ACTH}$, Adrenocorticotrophic hormone; BNP, B-type natriuretic peptide, $m / z$, mass-to-charge ratio 
Supplemental Table S1 Independent prediction abilities of traditional cardiac risk markers using multivariate Cox survival analyses for outcomes of death/MI, death/HF and MACE at 6 months, 1 year and 2 years in a subset of samples $(n=617)$ including molecular forms of B-type natriuretic peptide (BNP), BNP or N-terminal proBNP (NT-proBNP).

\begin{tabular}{llll}
\hline & Death/MI & Death/HF & MACE \\
\hline BNP & & & \\
6 months & $2.07(1.08-3.98), \mathrm{P}=0.029$ & $2.69(1.41-5.11), \mathrm{P}=0.003$ & $1.98(1.14-3.43), \mathrm{P}=0.015$ \\
1 year & $2.12(1.20-3.72), \mathrm{P}=0.009$ & $2.64(1.49-4.70), \mathrm{P}=0.001$ & $2.12(1.30-3.47), \mathrm{P}=0.003$ \\
2 years & $1.98(1.17-3.33), \mathrm{P}=0.010$ & $2.77(1.62-4.74), \mathrm{P}<0.001$ & $2.08(1.32-3.28), \mathrm{P}=0.002$ \\
BNP 5-32 & & & \\
6 months & $2.65(1.39-5.07), \mathrm{P}=0.003$ & $2.26(1.22-4.19), \mathrm{P}=0.010$ & $2.03(1.17-3.50), \mathrm{P}=0.011$ \\
1 year & $2.27(1.30-3.98), \mathrm{P}=0.004$ & $2.26(1.30-3.92), \mathrm{P}=0.004$ & $1.96(1.20-3.18), \mathrm{P}=0.007$ \\
2 years & $2.22(1.32-3.74), \mathrm{P}=0.003$ & $2.48(1.48-4.15), \mathrm{P}=0.001$ & $2.07(1.32-3.27), \mathrm{P}=0.002$ \\
BNP 4-32 & & & \\
6 months & $2.11(1.24-3.58), \mathrm{P}=0.006$ & $2.01(1.20-3.34), \mathrm{P}=0.008$ & $1.78(1.16-2.75), \mathrm{P}=0.009$ \\
1 year & $1.92(1.23-3.01), \mathrm{P}=0.004$ & $2.00(1.26-3.16), \mathrm{P}=0.003$ & $1.83(1.25-2.70), \mathrm{P}=0.002$ \\
2 years & $1.78(1.18-2.69), \mathrm{P}=0.006$ & $2.01(1.31-3.09), \mathrm{P}=0.001$ & $1.76(1.23-2.52), \mathrm{P}=0.002$ \\
BNP 3-32 & & & \\
6 months & $1.86(1.10-3.15), \mathrm{P}=0.022$ & $2.18(1.30-3.65), \mathrm{P}=0.003$ & $1.80(1.16-2.78), \mathrm{P}=0.009$ \\
1 year & $1.69(1.08-2.66), \mathrm{P}=0.022$ & $2.09(1.31-3.33), \mathrm{P}=0.002$ & $1.75(1.19-2.59), \mathrm{P}=0.005$ \\
2 years & $1.55(1.02-2.34), \mathrm{P}=0.039$ & $2.20(1.42-3.40), \mathrm{P}<0.001$ & $1.74(1.21-2.50), \mathrm{P}=0.003$ \\
NT-proBNP & & & \\
6 months & $1.58(0.91-2.75), \mathrm{P}=0.107$ & $2.35(1.25-4.40), \mathrm{P}=0.008$ & $1.63(1.01-2.63), \mathrm{P}=0.045$ \\
1 year & $1.45(0.91-2.30), \mathrm{P}=0.118$ & $2.49(1.42-4.37), \mathrm{P}=0.001$ & $1.68(1.10-2.56), \mathrm{P}=0.016$ \\
2 years & $1.45(0.94-2.23), \mathrm{P}=0.092$ & $2.50(1.49-4.20), \mathrm{P}=0.001$ & $1.64(1.11-2.43), \mathrm{P}=0.014$ \\
\hline \multicolumn{1}{r}{$\mathrm{BNP}, \mathrm{B}$ type } & &
\end{tabular}

BNP, B-type natriuretic peptide; HF, heart failure; MACE, major adverse cardiac event; MI, myocardial infarction; NT-proBNP, N-terminal pro B-type natriuretic peptide 